\title{
An infectious disease perspective - is destination therapy a viable option for total artificial hearts?
}

\author{
Rachel Zabner ${ }^{1}$, Lee D. Lam ${ }^{2}$, Carmelita Runyan ${ }^{2}$ \\ ${ }^{1}$ Department of Infectious Disease, ${ }^{2}$ Cedars Sinai Comprehensive Transplant Center, Cedars Sinai, Los Angeles, CA, USA \\ Correspondence to: Rachel Zabner, MD. Department of Infectious Disease, Cedars Sinai, 8700 Beverly Blvd, Room 465 W, Los Angeles, CA 90048, \\ USA. Email: Rachel.Zabner@cshs.org.
}

Submitted Nov 26, 2019. Accepted for publication Jan 21, 2020.

doi: $10.21037 / a c s .2020 .02 .07$

View this article at: http://dx.doi.org/10.21037/acs.2020.02.07

As the number of patients with end-stage heart failure requiring advanced therapy continues to grow, and the availability of donor organs for heart transplantation remains limited, the use of mechanical circulatory support devices (MCSDs) as a bridge to heart transplantation has increased dramatically. Currently, total artificial heart (TAH) devices are FDA-approved only for use in end-stage biventricular heart failure as a bridge to heart transplantation and are undergoing clinical trials for destination therapy (DT) (1).

There are a subset of patients awaiting orthotopic heart transplantation who are at high risk because they are highly sensitized and in need of desensitization protocols. Currently, patients may receive immunotherapy (biologics), intravenous immunoglobulins, and steroids. These treatments increase the risk of infection following desensitization. In addition, this subset includes patients with multiple sternotomies, prior heart transplant, and other conditions that pose a high risk, which may then preclude moving forward with heart transplantation. This raises the question, can TAH devices be offered as an alternative option for DT in these patients?

Although the published literature regarding infections pertains mainly to left ventricular assist devices (LVADs), the risk of infections applies to all implantable MCSDs that have drivelines or cannulas. As with other MCSDs, infections in TAHs are common but manageable, and do not directly increase morbidity or mortality rates whilst being used to bridge to transplantation (2).

In our experience at Cedars Sinai Medical Center (CSMC), of the 96 patients who received TAHs $\left(\right.$ SynCardia $\left.{ }^{\circledR}\right)$, the morbidity and mortality rate due to infections has been low. The infections found have been similar with percutaneous drivelines, including pulsating and continuous flow devices, as based on published data (3). The incidence of driveline infection at CSMC is $8 \%$. Infections in TAHs are classified as devicerelated and non-device related. The TAH device-related infections are more common in cannulas and drivelines. The etiology of early and late infections is predominantly bacterial. Most pathogens are gram positive bacteria, including Staphylococcus aureus, Staphylococcus epidermidis, and Enterococcus species, which colonize the skin and adhere to the implanted device, creating a biofilm. Infections with Pseudomonas are less common but difficult to treat. They usually occur after 30 days from implantation of the device. In contrast, fungal infections are uncommon.

The International Society for Heart and Lung Transplantation (ISHLT) published a consensus on Mechanical Circulatory Support (MCS) infection management and prevention strategies (4). Infectious complications are preventable with preoperative methicillin-resistant Stapbylococcus aureus/methicillinsensitive Stapbylococcus aureus (MRSA/MSSA) nasal swab screening and decolonization protocol. At the 2019 ISHLT conference, we presented data from the CSMC antibiotic selection for surgical prophylaxis protocol, showing no infections in the first 30 days. Published data for 1-year driveline infections for TAHs were not invasive and only $4 \%$ had mediastinitis (5). In our experience, despite having an open chest for $24-48$ hours post-TAH implant, there were no reported mediastinal infections. We have proposed that patients with prior history of MRSA/MSSA positive screens have surveillance nasal swabs. If the swab is positive, 
we recommend repeating the decolonization protocol. This would potentially prevent driveline infections further, after 30 days.

Non-device related infections are the largest category of MCSD infection (6). While reviewing our data, an interesting finding emerged, with respect to increased incidence of acute cholecystitis. At CSMC, the incidence of acute cholecystitis in the TAHs population was $18 \% v s$. $10 \%$ for LVADs. However, this did not lead to increased mortality, due to proactive surveillance of liver function tests, ultrasound scans, and hepatobiliary iminodiacetic acid (HIDA) scans, which led to early intervention with laparoscopic cholecystectomy or cholecystostomy.

In our experience, patients who had infectious complications secondary to prior malignancies such as multiple myeloma or other patients with amyloidosis, sarcoidosis or other immunosuppressive states, have a higher risk for opportunistic infections after heart transplantation. These can be more difficult to prevent and can sometimes be fatal once the cascade of additional immunosuppressants are added, along with antithymocyte globulin, biologics, steroids, and calcineurin inhibitors. Some potential infections seen include atypical mycobacterium, aspergillosis, cytomegalovirus (CMV) reactivation causing disease, and reactivation of Chagas disease (7). These infectious risks contribute to increasing morbidity and mortality rates after transplantation. In our opinion, these patients would potentially have had a better outcome, with lower incidence of infectious complications, if they were to be identified early and selected for TAHs as DT instead of as a bridge to transplantation. Data from a small number of patients shows a 100\% 5-year survival rate after implantation of their original TAH device (8). Currently, there are ongoing DT trials of TAHs in the US and Europe (1).

As technology advances, more efficient and better designed drivers are being developed for TAHs, such as SynCardia ${ }^{\circledR}$, Carmat $^{\circledR}$ and BiVicor ${ }^{\circledR}$. Future models will have a simpler design, will be more responsive to patient's physiological needs, and will be totally implantable, which may eliminate the pneumatic driveline. In addition, longer battery life, lower power consumption and changes in transcutaneous technology will go a long way towards reducing infections. Such devices are under investigation in Europe and the US (9), and more will likely follow, offering the potential to make TAH in DT a more viable alternative for heart failure patients.

\section{Acknowledgments}

We would like to thank the Mechanical Circulatory Support team at Cedars Sinai Medical Center.

\section{Footnote}

Conflicts of Interest: The authors have no conflicts of interest to declare.

\section{References}

1. SynCardia 70cc TAH-t for Destination Therapy (DT) (RA-540). Available online: https://clinicaltrials.gov/ct2/ show/NCT02232659

2. Hidalgo LF, Shah KB, Cooke RH, et al. Infections in Patients with a Total Artificial Heart Are Common but Rarely Fatal. ASAIO J 2017;63:736-9.

3. Kirklin JK, Naftel DC, Pagani FD, et al. Seventh INTERMACS annual report: 15,000 patients and counting. J Heart Lung Transplant 2015;34:1495-504.

4. Kusne S, Mooney M, Danziger-Isakov L, et al. An ISHLT consensus document for prevention and management strategies for mechanical circulatory support infection. J Heart Lung Transplant 2017;36:1137-53.

5. Torregrossa G, Morshuis M, Varghese R, et al. Results with SynCardia total artificial heart beyond 1 year. ASAIO J 2014;60:626-34.

6. Hannan MM, Xie R, Cowger J, et al. Epidemiology of infection in mechanical circulatory support: A global analysis from the ISHLT Mechanically Assisted Circulatory Support Registry. J Heart Lung Transplant 2019;38:364-73.

7. Sandkovsky U, Florescu DF, Um JY, et al. Cytomegalovirus reactivation and colitis after left ventricular assist device placement. Int J Infect Dis 2013;17:e348-51.

8. Arabía FA, Cantor RS, Koehl DA, et al. Interagency registry for mechanically assisted circulatory support report on the total artificial heart. J Heart Lung Transplant 2018;37:1304-12.

9. Carmat TAH Early Feasibility Study. Available online: https://clinicaltrials.gov/ct2/show/NCT04117295

Cite this article as: Zabner R, Lam LD, Runyan C. An infectious disease perspective-is destination therapy a viable option for total artificial hearts? Ann Cardiothorac Surg 2020;9(2):126-127. doi: 10.21037/acs.2020.02.07 